\title{
THE NIJMEGEN HIGH FIELD MAGNET LABORATORY
}

\author{
J.A.A.J. PERENBOOM and K. VAN HULST \\ High Field Magnet Laboratory, University of Nijmegen, Toernooiveld, NL-6525 ED Nijmegen. The Netherlands
}

\begin{abstract}
The Nijmegen high field facility is configured around a 6-MW electrical power supply and the associated water-cooling system. Five magnet stations are available: three water-cooled magnets, with a maximum field of $20 \mathrm{~T}$. and two hybrid magnet systems; our latest hybrid. Nijmegen-II, generates fields up to $30.4 \mathrm{~T}$ in a $32-\mathrm{mm}$ bore
\end{abstract}

\section{Introduction}

The University of Nijmegen has been operating its facility to generate high static magnetic fields since 1976. The Nijmegen High Field Magnet Laboratory (NHFML) is housed on two floors of the physics wing at the Faculty of Science. The basic infrastructure of the facility consists of an electrical power supply, which presently delivers $6 \mathrm{MW}$ and can be extended to $9 \mathrm{MW}$, and a closed-cycle water cooling system.

The main effort of the in-house scientists is in magneto-optical and magneto-transport studies of semiconductors; current research programmes include the study of critical properties of superconducting materials, specific heat and de Haasvan Alphen spectroscopy of heavy-Fermion systems, magnetisation of ferromagnetic materials, and magnetic separation. The NHFML is strongly committed to international collaborative research programmes. It operates as a user-oriented facility: the high field facility, and the associated cryogenic, spectroscopic and data-acquisition equipment, is available for all qualified academic research.

\section{The installation}

The de current to encrgise the magnets is provided by two 3-MW power supplies $(300 \mathrm{~V} \times$ $10 \mathrm{kA}$ ), which are usually operated in parallel. Each unit consists of a transformer (with induction control), diode rectifiers and a passive LC- filter. Further regulation is provided by a transistor bank of 2000 germanium transistors in parallel. A hydraulic system will set the induction control and so realise coarse control of the current. The voltage across the transistor bank is used for fine control, and may be varied rapidly for the purpose of accurately regulated magneticfield sweeps or magnetic-field modulation. The reference for the power supplies is set by means of a remote panel controlled by the experimenter and may represent any time-profile of the magnetic field within certain limits.

The cooling of the resistive magnets and the electrical power supplies is achieved with a closed-cycle water-cooling circuit. Fig. 1 shows the general layout of the installation, indicating a magnet station, the 6-MW power supply, and the cooling system. A maximum flow of $400 \mathrm{~m}^{3} / \mathrm{h}$ of deionised water is pressed through the magnet coils at a pressure of up to 23 bar. The cooling water is kept cool through its contact with a mass of 150 tons of ice in a $450 \mathrm{~m}^{3}$ basin, frozen using a compressor-driven refrigerator cooled with ground water. This mass of ice and cold water represents a cold buffer of $18 \mathrm{MWh}$.

The NHFML operates (with certain restrictions on the average energy consumption) on work-days starting after $13.00 \mathrm{~h}$, except during four weeks in December; the nominal power of 6.3 MW is available without any restrictions on workdays from $23.00 \mathrm{~h}$ to $8.00 \mathrm{~h}$, and at weekends around the clock. On a typical day two shifts of nominally four hours will be scheduled, although regularly very long sessions lasting until the early morning will take place. 


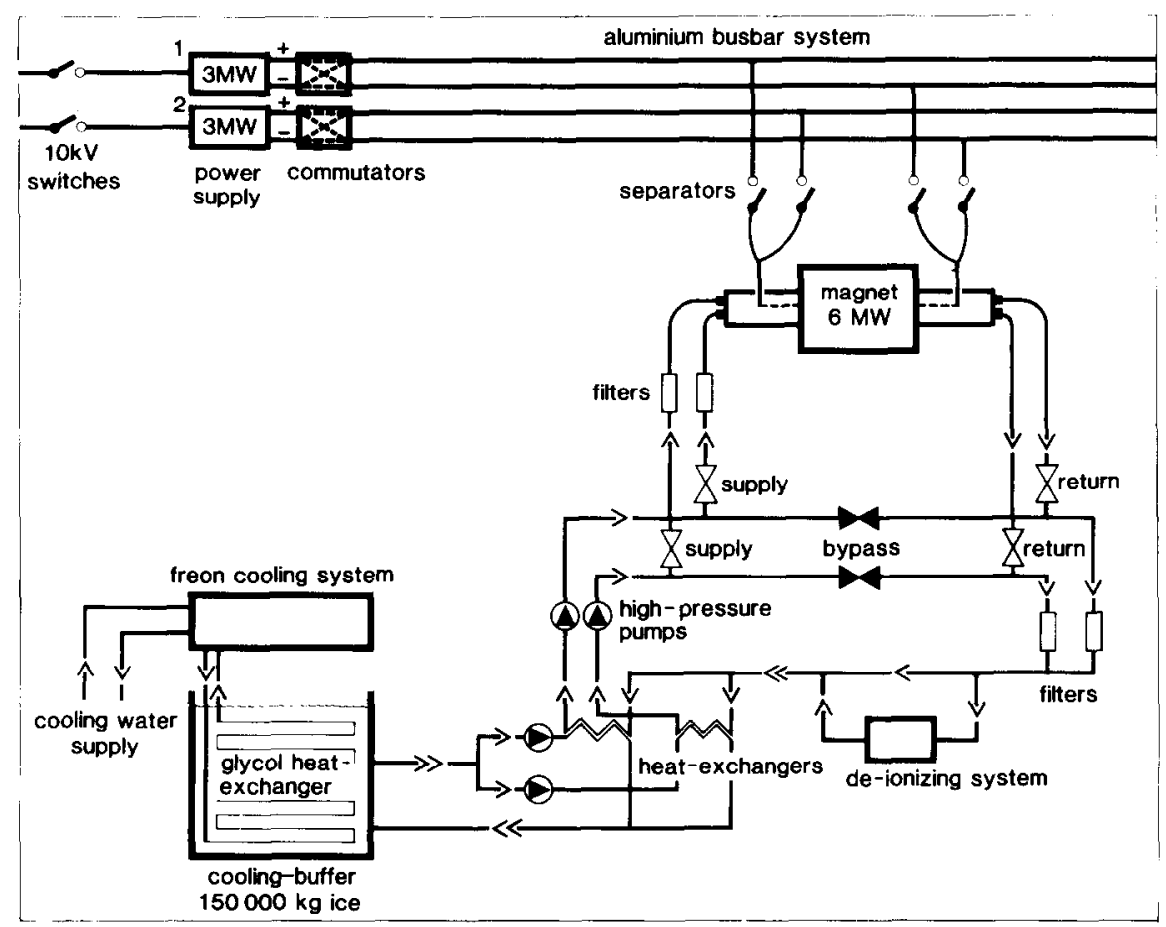

Fig. 1. Schematic representation of the installation at the NHFML, indicating a magnet station, the 6-MW electrical power supply, and the closed-cycle water-cooling system with $18-\mathrm{MWh}$ cold buffer.

\section{The high field magnets}

The NHFML has five magnet stations operational, the present configuration is listed in table I together with some specifications of the magnets. The magnets are being run and maintained by the laboratory staff. There is no programme in Nijmegen to develop and construct magnets, however. The hybrid magnet systems Nijmegen-I and Nijmegen-II have been built under a collaborative contract at M.I.T. in Cambridge, Massachusetts. Nijmegen-I has been in operation since 1978 and will soon be outfitted

Table I

Magnet stations at the NHFML

\begin{tabular}{lll}
\hline Magnet system & $B_{\max }(\mathrm{T})$ & Bore $(\mathrm{mm})$ \\
\hline 1. Hybrid magnet N-I & 25.4 & 32 \\
2. Bitter coil & 15.2 & 60 \\
3. Bitter coil & 14.8 & 53 \\
4. Duplex Bitter coil & 20.0 & 32 \\
5. Hybrid magnet N-II & 30.4 & 32 \\
\hline
\end{tabular}

with a $6.0-\mathrm{MW}, 53-\mathrm{mm}$ bore insert coil. The second, more advanced hybrid magnet system has been in operation since early 1987. The design of the 30-T magnet, the features of the subcooled superfluid helium cryostat and the first testing at M.I.T. and at Nijmegen, have been reported earlier [1-4]. A cross-section of the 30-T hybrid magnet system is shown in fig. 2 . The NbTi superconductive coil has been designed for operation at $1.8 \mathrm{~K}$ and it will then generate $11 \mathrm{~T}$ at $2000 \mathrm{~A}$ (operation at $4.2 \mathrm{~K}$ is also possible: $8.3 \mathrm{~T}$ at $1500 \mathrm{~A}$ ). The insert consists of two concentric radially-cooled Bitter coils. For their compression they rely mainly on a set of iron armatures at either end of the stacks, which also contribute to the total magnetic field. The cooling of the radiation shield and the precooling of the supcrconductive coil down to about $15 \mathrm{~K}$ is accomplished by a Philips Cryogenerator, as with the 25-T hybrid magnet, Nijmegen-I [5]. Cooldown from room temperature to $15 \mathrm{~K}$ takes about $200 \mathrm{~h}$. It then takes 


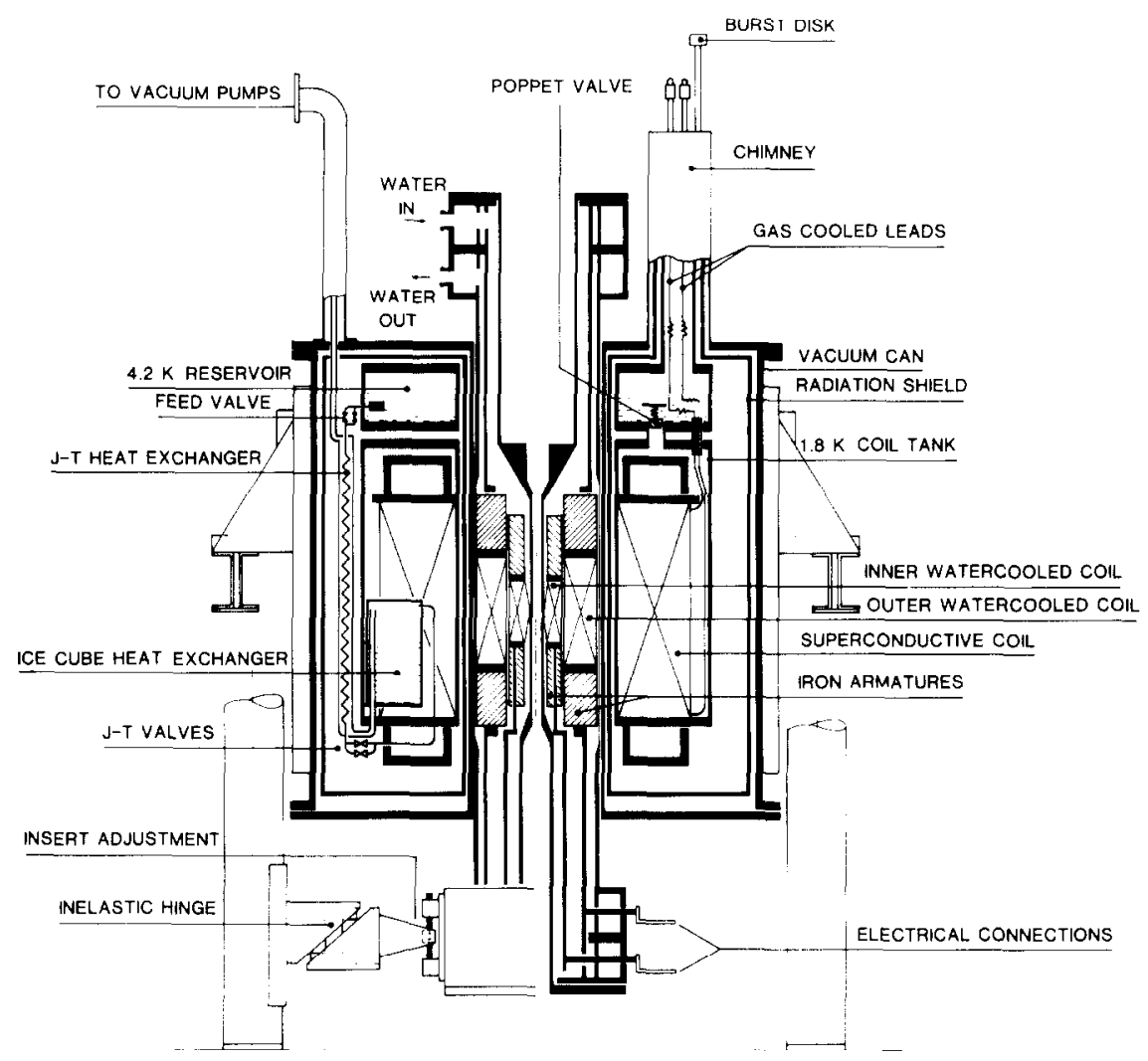

Fig. 2. Cross-section of the 30-T hybrid magnet system, Nijmegen-II. The system includes a NbTi superconductive outer coil, operated at $1.8 \mathrm{~K}$ in an atmospheric-pressure, sub-lambda cooled helium bath, and two concentric radially-cooled Bitter coils.

about $90 \mathrm{~min}$ to fill the coil tank and the reservoir with liquid helium. While there is liquid helium in the cryostat, the cryogenerator is kept running to provide cooling for the $80-\mathrm{K}$ radiation shield. Another $75 \mathrm{~min}$ are required for cooling the coil tank from $4.2 \mathrm{~K}$ to $1.8 \mathrm{~K}$, using the cooling power of two $1250 \mathrm{~m}^{3} / \mathrm{h}$ vacuum pumps. The total amount of liquid helium required for filling and cooldown, inclusive of transfer losses and boil-off, is about $550 \mathrm{l}$.

\section{Nijmegen-II}

During performance tests [4] we found that the helium boil-off became excessive when the coils were energised. In the original cryostat design, the cold mass was suspended from the cryostat top cover by means of six steel cables attached to the bottom end of the coil tank. Centricity of the suspension points at both ends and adjustment of the cables to equal lengths would take account of proper alignment, and of centricity of the field axis with the water-cooled insert coils. Fibreglass straps at the bottom end of the coil tank were used to secure its radial and rotational position. These straps, together with the weight of the cold mass, would suffice to withstand the interaction forces between insert and superconducting coil under normal operating conditions.

Six heavy members, mounted on the bottom cover of the cryostat and adjustable from the outside, would act as "stops" so as to cope with excessive forces (e.g. those occurring during an insert failure). They would limit the vertical 
movement of the cold mass to $1 \mathrm{~mm}$ in either direction and so protect the suspension structure from being overstressed. The stops, when centred, could travel approximately $1 \mathrm{~mm}$ in either direction before touching the cold mass. During first tests at Nijmegen, the position of the coil tank within the cryostat was found to be less stable than we had hoped: at moderately high combined fields of insert-coils and superconductive magnet, we had to readjust the setting of these stops to prevent, or at least reduce, thermal shorts at the higher ficld strengths. Shortly after the magnet system was made available for physics research, the situation worsened to the point where $1.8 \mathrm{~K}$ operation was no longer possible. By itself this was enough of a reason to dismantle the cryostat, but problems with degrading contacts to the gas-cooled current leads at the cold end and unsatisfactory efficiency of the helium transfer made the decision easier.

Based on an idea of M.J. Leupold of M.I.T., one of the original designers of this hybrid magnet system, we designed fibreglass struts to rigidly support the cold mass from the bottom cover of the cryostat. They will replace the six "stops" as the struts will withstand axial as well as rotational forces under all anticipated conditions. The maximum radial forces that might occur, however, are close to the limits and we therefore added six radial straps for additional support in that direction. The struts are $180 \mathrm{~mm}$ long dogbone-shaped members. Their ends are clamped to the bottom cover and the bottom of the coil tank. Each strut consists of a package of ten $25-\mathrm{mm}$ wide and $3-\mathrm{mm}$ thick elements, so that stiffness in one sideways direction is combined with flexibility in the other. The struts are mounted so that they are flexible in the radial direction and thermal contraction of the cold mass will cause them to become slightly bent into an
S-shape without introducing any significant stress, while radial and rotational forces on the cold mass will be met by the full stiffness of part or all of the struts, respectively. The spring constant in the radial direction of the six struts together was estimated to be about $3 \times$ $10^{4} \mathrm{~N} / \mathrm{mm}$. Measurements indicate that it is actually about $25 \%$ higher.

To keep the heat input into the $1.8 \mathrm{~K}$ space as low as possible, the struts as well as the radial straps have been heat-sunk to the radiation shield at their mid-lengths. The extra heat-load on the radiation shield amounts to about $5 \mathrm{~W}$, which is almost negligible with respect to the cryogenerator's cooling power at $80 \mathrm{~K}$. The estimated heat input into the coil tank through the new suspension parts will be approximately equal to that of the parts that have been removed. We therefore expect no significant change in the standing loss of our hybrid magnet system, which with only $6 \mathrm{~W}$ was most satisfactory in the earlier tests [4]. We expect that the present modification will result in a considerable reduction of liquid helium consumption under operating conditions, and anticipate that as of the end of 1988 this $30-\mathrm{T}$ magnet system will be routinely available for scientific research.

\section{References}

[1] M.J. Leupold, Y. Iwasa and R.J. Weggel, J. Physique 45 (1984) C1-41.

[2] M.J. Leupold and Y. Iwasa, Cryogenics 26 (1986) 579.

[3] M.J. Leupold, Y. Iwasa, J.R. Hale, R.J. Weggel and K. van Hulst, in: Magnet Technology. MT-9, C. Marinucci and P. Weymuth, eds. (SIN, Villigen, 1985), p. 215.

[4] K. van Hulst and J.A.A.J. Perenboom, IEEE Trans. Magn. MAG-24 (1988) 1397.

[5] K. van Hulst, IEEE Trans. Magn. MAG-17 (1981) 1790. 